\title{
HYPERBOLIC BIRKHOFF CENTERS
}

BY

I. P. MALTA

\begin{abstract}
The purpose of this paper is to show that if $f$ is a diffeomorphism of a compact manifold whose Birkhoff center, $c(f)$, is hyperbolic and has no cycles, then $f$ satisfies Axiom $A$ and is $\Omega$-stable. To obtain a filtration for $c(f)$, the concept of an isolated set for a homeomorphism of a compact metric space is introduced. As a partial converse it is proved that if $c(f)$ is hyperbolic and $f$ is $\Omega$-stable, then $c(f)$ has the no cycle property. A characterization of $\Omega$-stability when $c(f)$ is finite is also given.
\end{abstract}

Introduction. In this paper we consider a diffeomorphism $f$ of a compact manifold $M$ whose Birkhoff center is hyperbolic. We prove that if such a diffeomorphism $f$ has no cycles, then it satisfies Axiom A and is $\Omega$-stable.

We denote by $\Omega=\Omega(f)$ the set of nonwandering points of $f$. Smale's Axiom A requires

(a) $\Omega$ has a hyperbolic structure.

(b) The periodic points are dense in $\Omega$.

In Smale's paper about $\Omega$-stability [9], he proved that if $f$ satisfies Axiom $\mathrm{A}$ and has no cycles, then $f$ is $\Omega$-stable (for an easier proof see [6]). Later on, Newhouse [5] showed that if the closure of the $\alpha$-limit set of $f$, denoted by $L^{-}$, is hyperbolic and does not admit cycles, then $f$ satisfies Axiom A and has no cycles, and therefore $f$ is $\Omega$-stable. In this paper we obtain the same conclusions by requiring the condition of hyperbolicity and the property of having no cycles for a subset of $\mathrm{L}^{-}$, the Birkhoff center of $f$, denoted by $c(f)$. We define $c(f)$ to be the closure of the set $\{x \in M \mid x \in \alpha(x) \cap \omega(x)\}$, where $\alpha(x)$ and $\omega(x)$ are the $\alpha$-limit and $\omega$-limit sets of $x$ for $f$. Our main result is the following theorem.

Theorem B. If $c(f)$ is hyperbolic and has no cycles, then $f$ satisfies Axiom A and has no cycles. In particular $f$ is $\Omega$-stable.

We also prove, following [7], a partial converse of this result.

THeOREM C. If $c(f)$ is hyperbolic and $f$ is $\Omega$-stable, then $c(f)$ has the no cycle property. In particular, $c(f)=\Omega(f)$.

In order to prove Theorem $\mathrm{B}$, we initially consider a homeomorphism $f$ of a compact metric space $K$. We introduce some concepts which generalize others used in the study of a diffeomorphism of a compact manifold. In this context, the basic concept introduced is the one of isolated set for a homeomorphism. As we show in Proposition (1.2), isolated sets have a fundamental property valid, in the case of a diffeomorphism, for hyperbolic sets with local product structure: if there exists a

Received by the editors November 11, 1978 and, in revised form, June 5, 1979.

AMS (MOS) subject classifications (1970). Primary 58F15, 58F20. 
decomposition having no cycles, we may obtain a filtration for the elements of this decomposition. We show then that if $c(f)$ is isolated and satisifies the above hypothesis, then $c(f)$ coincides with the nonwandering set of $f, \Omega(f)$. More explicitly, we say that a compact set $\Lambda \subset K$ is isolated for $f$ if it is invariant by $f$ (i.e., $f(\Lambda)=\Lambda$ ) and there exists a neighborhood $U$ of $\Lambda$ such that $\Lambda$ is the maximal invariant set for $f$ in $U$, that is, $\cap_{n \in \mathbf{Z}} f^{n}(U)=\Lambda$. We then prove the following theorem.

THEOREM A. If $c(f)$ is an isolated set for $f$ and admits a decomposition $c(f)=\Lambda_{1}$ $\cup \cdots \cup \Lambda_{l}$ into isolated sets having no cycles, then $c(f)=\Omega(f)$.

From this theorem we prove the main result of the paper, Theorem B, as stated above. The idea of the proof is as follows. Let $f$ be a $C^{r}$ diffeomorphism, $r \geqslant 1$, of a compact $C^{\infty}$ manifold without boundary, whose Birkhoff center $c(f)$ is hyperbolic. From Anosov's Closing Lemma we can show that $c(f)$ coincides with the closure of the set of periodic points of $f$, denoted by $\bar{P}$. Then using Newhouse's theorem [5] relative to the decomposition of $\bar{P}$ into basic sets, we obtain a decomposition of $c(f)$. Theorem B then follows, showing that this decomposition satisfies the hypothesis of Theorem A.

In $\S 1$ we establish some notation and introduce the definitions in the context of compact metric spaces. In particular, we define an isolated set for a homeomorphism of a compact metric space, which generalizes the idea of hyperbolic sets having local product structure for a diffeomorphism of a compact manifold. In analogy to the invariant manifolds of a hyperbolic set [3], we associate invariant spaces to an isolated set. We obtain, in this context, well-known results for diffeomorphisms, including a filtration lemma, that we use in the proof of Theorem A.

In $\$ 2$ we restrict our considerations to diffeomorphisms of a $C^{\infty}$ compact manifold. We restate the definition of a hyperbolic set having local product structure for a diffeomorphism and recall some known results. These results enable us to show that such sets are isolated. Also the invariant manifolds coincide with the invariant spaces as defined in $\$ 1$. We prove Theorem B making use of Anosov's Closing Lemma, Newhouse's theorem for the decomposition of $\bar{P}$ and Theorem A.

In $\$ 3$ we prove Theorem $C$. For this purpose, we introduce the concept of $\bar{P}$-stability; that is, the stability of the closure of the set of periodic points of a diffeomorphism $f$. We prove that if $\bar{P}=\overline{\operatorname{Per}(f)}$ is hyperbolic and $f$ is $\bar{P}$-stable, then $\bar{P}$ has the no cycle property. This result is similar to the one known as $\Omega$-explosion. In fact, the proof of the above result is essentially the same given by Palis in [7].

We observe that to prove our main result, we need to consider the restriction of a diffeomorphism to certain invariant subsets of the ambient manifold, which do not have in general a differentiable structure. This is the main reason to study isolated sets for homeomorphisms in the first part of the paper, where Theorem $A$ is proved.

We also point out that Theorem $B$ implies a positive answer to a question posed by Newhouse in [5]. 
This work corresponds to my doctoral thesis at IMPA, under the guidance of $\mathbf{J}$. Palis. I wish to thank him, R. Mañé and S. Newhouse for suggestions and encouragement.

1. Isolated sets. In this section we will define isolated sets for a homeomorphism of a compact metric space $K$, which generalize the idea of hyperbolic sets with local product structure of a diffeomorphism. In analogy to the invariant manifolds of a hyperbolic set for a diffeomorphism, we will associate two invariant spaces, called the unstable space and the stable space, to each isolated set for a homeomorphism. We can therefore consider the notion of cycles in a disjoint family of isolated sets.

The basic result of this section is Proposition (1.2). From it we obtain a Filtration Lemma (1.8) and Lemma (1.9) which will be used to prove Theorem A.

Here $f$ is a homeomorphism of a compact metric space $K$. We denote by $P$ the set of periodic points of $f$ and by $\Omega$ the set of nonwandering points of $f$. That is,

$$
\begin{aligned}
& \Omega=\left\{x \in K \mid \text { given a neighborhood } U \subset K \text { of } x \text { and } n_{0}>0,\right. \\
& \text { there exists } \left.n \in \mathbf{Z} \text { with }|n|>n_{0} \text { such that } f^{n}(U) \cap U \neq \varnothing\right\} .
\end{aligned}
$$

For a subset $D \subset K, \bar{D}$ will denote its closure in $K$, and int $D$ will denote its interior in $K$. For each $x \in K$, we denote by $\theta(x)$ the orbit of $x$, and by $\theta_{-}(x)$ and $\vartheta_{+}(x)$ the negative and positive orbit of $x$, respectively. Let $x, y \in K$. We say that $y \in \alpha(x)$ if there is a sequence of integers $n_{i} \rightarrow \infty$ such that $f^{-n_{i}}(x) \rightarrow y$ as $i \rightarrow \infty$. In a similar way, we define $\omega(x)$ to be the set of $y \in K$ such that there is a sequence of integers $n_{i} \rightarrow \infty$ such that $f^{n_{i}}(x) \rightarrow y$ as $i \rightarrow \infty$. The set $\alpha(x)$ is called the $\alpha$-limit set of $x$ and $\omega(x)$ is the $\omega$-limit set of $x$. Notice that $\alpha(x)$ (or $\omega(x)$ ) is closed, nonempty and invariant under $f$, that is, $f(\alpha(x))=\alpha(x)$ for every $x$ in $K$.

Definition. A compact subset $\Lambda \subset K$ is isolated for the homemorphism $f$ : $K \rightarrow K$ if it is invariant under $f$, that is $f(\Lambda)=\Lambda$, and if there is a neighborhood $U$ of $\Lambda$ such that $\cap_{n \in \mathbf{Z}} f^{n}(U)=\Lambda$.

In the sequel $\Lambda \subset K$ is assumed to be an isolated set for $f$. As in the case of hyperbolic sets for a diffeomorphism, we associate to an isolated set $\Lambda$, invariant spaces, $W^{s}(\Lambda)$ and $W^{u}(\Lambda)$, called the stable space and unstable space of $\Lambda$, respectively, defined as follows:

$$
W^{u}(\Lambda)=\{x \in K \mid \alpha(x) \subset \Lambda\} \quad \text { and } \quad W^{s}(\Lambda)=\{x \in K \mid \omega(x) \subset \Lambda\} .
$$

If $U$ is a compact neighborhood of $\Lambda$ such that $\bigcap_{n \in \mathbf{Z}} f^{n}(U)=\Lambda$, we define $W_{\text {loc }}^{s}(\Lambda, U)=\bigcap_{n>0} f^{-n}(U)$ and $W_{\text {loc }}^{u}(\Lambda, U)=\bigcap_{n>0} f^{n}(U)$. Thus we have $W^{s}(\Lambda)$ $=\bigcup_{n \geqslant 0} f^{-n}\left(W_{\mathrm{loc}}^{s}(\Lambda, U)\right), W^{u}(\Lambda)=\bigcup_{n \geqslant 0} f^{n}\left(W_{\mathrm{loc}}^{u}(\Lambda, U)\right)$ and $W_{\mathrm{loc}}^{s}(\Lambda, U) \cap$ $W_{\text {loc }}^{u}(\Lambda, U)=\Lambda$.

We consider in $W_{\mathrm{loc}}^{\sigma}(\Lambda, U), \sigma=s, u$, the topology induced by the metric in $K$.

Let $U$ be a compact neighborhood of $\Lambda$ such that $\bigcap_{n \in Z} f^{n}(U)=\Lambda$. If $V$ is a compact neighborhood of $\Lambda$ in $W_{\text {loc }}^{s}(\Lambda, U)$ such that $f(V) \subset V$, we call the set $D=\overline{V-f(V)}$ a fundamental domain for $W_{\text {loc }}^{s}(\Lambda, U)$. In a similar way we define a fundamental domain for $W_{\text {loc }}^{u}(\Lambda, U)$. We observe that if $x \in W^{s}(\Lambda)-\Lambda$, then there exists an integer $k$ such that $f^{k}(x) \in D$. If $D \cap \Lambda=\varnothing$, we call $D$ a proper fundamental domain. 
(1.1) Proposition. If $\Lambda$ is an isolated set for $f$, then

$$
D^{s}=\overline{W_{\text {loc }}^{s}(\Lambda, U)}-f\left(W_{\text {loc }}^{s}(\Lambda, U)\right)
$$

is a proper fundamental domain for $W_{\mathrm{loc}}^{s}(\Lambda, U)$.

Proof. We need only to prove that $\Lambda$ is contained in the interior of $f\left(W_{\text {loc }}^{s}(\Lambda, U)\right)$ in $W_{\text {loc }}^{s}(\Lambda, U)$. In fact, $f\left(W_{\text {loc }}^{s}(\Lambda, U)\right)=f(U) \cap W_{\text {loc }}^{s}(\Lambda, U)$, thus $\Lambda \subset f($ int $U) \cap W_{\text {loc }}^{s}(\Lambda, U) \subset f\left(W_{\text {loc }}^{s}(\Lambda, U)\right)$ and $f($ int $U) \cap W_{\text {loc }}^{s}(\Lambda, U)$ is an open neighborhood of $\Lambda$ in $W_{\text {loc }}^{s}(\Lambda, U)$. So we have $D^{s} \cap \Lambda=\varnothing$.

The following fact is a basic one for our study since it enables us to obtain several results, including a filtration lemma, which we will use in the proof of Theorem A. Notice that it generalizes an analogous result for hyperbolic sets with local product structure for a diffeomorphism [4].

(1.2) Proposition. Let $V$ be a neighborhood of $D^{s}$ in $K$. Then $U^{\prime}=W_{\text {loc }}^{u}(\Lambda, U) \cup$ $\vartheta_{+}(V)$ is a neighborhood of $\Lambda$ in $K$, where $\theta_{+} V=\cup_{n>0} f^{n}(V)$.

Proof. Let us suppose the statement is false. Then if $W$ is a neighborhood of $\Lambda$ in $K$, we have $W \varnothing U^{\prime}$, that is, there is a sequence $x_{n} \in U-U^{\prime}$ which converges to $x$ in $\Lambda$. Since $x_{n} \notin W_{\text {loc }}^{u}(\Lambda, U)=\cup_{n>0} f^{n}(U)$ we have $\theta_{-}\left(x_{n}\right) \not U$. For each $n$, let $m_{n} \geqslant 0$ be such that $f^{-k}\left(x_{n}\right) \in U$ for $0 \leqslant k \leqslant m_{n}$, but $f^{-\left(m_{n}+1\right)}\left(x_{n}\right) \notin U$. Thus $y_{n}=f^{-m_{n}}\left(x_{n}\right) \in U-f(U)$. We observe that $m_{n} \rightarrow \infty$ as $n \rightarrow \infty$ since $\Lambda$ is compact, $f$-invariant, and $x_{n} \rightarrow \Lambda$. We can assume that $y_{n}$ converges to $y \in U$. We now claim that $y \in W^{s}(\Lambda)-\Lambda$. It is clear that $y \notin \Lambda$ since $\Lambda \subset$ int $f(V)$, thus all we need to prove is $y \in W^{s}(\Lambda)$. But if $y \notin W^{s}(\Lambda)$ there exists $k>0$ such that $f^{k}(y) \notin U$, so for $n$ sufficiently large we have $f^{k}\left(y_{n}\right)=f^{-m_{n}+k}\left(x_{n}\right) \notin U$. Since $m_{n} \rightarrow \infty$ as $n \rightarrow \infty, 0<m_{n}-k \leqslant m_{n}$ for $n$ sufficiently large, which gives a contradiction. Thus $y \in W^{s}(\Lambda)$ and we can choose $k_{0} \in Z$ such that $f^{k_{0}}(y) \in D^{s}$. Then $f^{k_{0}}\left(y_{n}\right)=f^{-m_{n}+k_{0}}\left(x_{n}\right) \in V$ when $n$ is large and so $x_{n} \in U^{\prime}$ contradicting our assumption.

(1.3) Lemma. Let $\Lambda_{1}, \Lambda_{2} \subset K$ be isolated sets for $f$ such that $\Lambda_{1} \cap \Lambda_{2}=\varnothing$. Then

(i) $\overline{W^{u}}\left(\Lambda_{1}\right) \cap W^{s}\left(\Lambda_{2}\right) \neq \varnothing \Rightarrow \overline{W^{u}}\left(\Lambda_{1}\right) \cap \hat{W}^{s}\left(\Lambda_{2}\right) \neq \varnothing$,

(ii) $\overline{W^{u}}\left(\Lambda_{1}\right) \cap \hat{W}^{u}\left(\Lambda_{2}\right) \neq \varnothing \Rightarrow \overline{W^{u}}\left(\Lambda_{1}\right) \cap \hat{W}^{s}\left(\Lambda_{2}\right) \neq \varnothing$

where $\overline{W^{\circ}}\left(\Lambda_{i}\right)$ is the closure of $W^{o}\left(\Lambda_{i}\right)$, and $\hat{W}^{\sigma}\left(\Lambda_{i}\right)=W^{o}\left(\Lambda_{i}\right)-\Lambda_{i}$ for $i=1,2$ and $\boldsymbol{\sigma}=s, u$.

Proof. Let $U$ be a compact neighborhood of $\Lambda_{2}$ such that $\bigcap_{n \in Z} f^{n}(U)=\Lambda_{2}$, and let $D^{s}=D^{s}\left(\Lambda_{2}, U\right)$ be a proper fundamental domain for $W_{\text {loc }}^{s}\left(\Lambda_{2}, U\right)$.

(i) Let $x_{n} \in W^{u}\left(\Lambda_{1}\right)$ such that $x_{n} \rightarrow x \in \overline{W^{u}}\left(\Lambda_{1}\right) \cap \Lambda_{2}$, and let $F=\overline{\cup_{n>0} \mathcal{O}\left(x_{n}\right)}$. Then $F \subset \overline{W^{u}}\left(\Lambda_{1}\right)$ and so we need only to prove that $F \cap D^{s} \neq \varnothing$.

Given any neighborhood $V$ of $D^{s}$, with $V \cap \Lambda_{2}=\varnothing$, let $U^{\prime}=W_{\text {loc }}^{u}\left(\Lambda_{2}, U\right) \cup$ $\vartheta_{+}(V)$ and $k \geqslant 0$ such that $x_{k} \in U^{\prime}$. Since $x_{k} \notin W^{u}\left(\Lambda_{2}\right)$ (recall we have $x_{k} \in$ $\left.W^{u}\left(\Lambda_{1}\right)\right)$ there is $n_{k} \geqslant 0$ such that $x_{k} \in f^{n_{k}}(V)$. Thus $\cup_{n>0} \theta\left(x_{n}\right) \cap V \neq \varnothing$ for every $V$, which implies $F \cap D^{s} \neq \varnothing$.

(ii) If $x \in \overline{W^{u}}\left(\Lambda_{1}\right) \cap \hat{W}^{u}\left(\Lambda_{2}\right)$ then $\varnothing \neq \alpha(x) \subset \overline{W^{u}}\left(\Lambda_{1}\right) \cap \Lambda_{2}$. Thus $\overline{W^{u}}\left(\Lambda_{1}\right) \cap W^{s}\left(\Lambda_{2}\right) \neq \varnothing$ and from (i) we have $\overline{W^{u}}\left(\Lambda_{1}\right) \cap \hat{W}^{s}\left(\Lambda_{2}\right) \neq \varnothing$. 
Let $\Lambda_{1}, \Lambda_{2}, \ldots, \Lambda_{l} \subset K$ be disjoint isolated sets for $f$.

Definition. A cycle for the family $\left\{\Lambda_{i}\right\}_{i=1, \ldots, l}$ is a sequence $\Lambda_{i}, \ldots, \Lambda_{i_{k}}$ such that $\Lambda_{i_{1}}=\Lambda_{i_{k}}$ and $\hat{W}^{u}\left(\Lambda_{i_{j}}\right) \cap \hat{W}^{s}\left(\Lambda_{i_{j+1}}\right) \neq \varnothing$ for $1 \leqslant j<k$.

REMARK. The sequence $\Lambda_{i}, \ldots, \Lambda_{i_{k}}=\Lambda_{i_{1}}$ is a cycle if there are points $x_{1}, \ldots, x_{k-1}$ such that $x_{j} \notin \cup_{m=1}^{k} \Lambda_{i_{m}}, \alpha\left(x_{j}\right) \subset \Lambda_{i_{j}}$ and $\omega\left(x_{j}\right) \subset \Lambda_{i_{j+1}}$ for $1 \leqslant j<k$.

(1.4) LEMMA. Let $\left\{\Lambda_{1}, \ldots, \Lambda_{l}\right\}$ be a family of disjoint isolated sets for $f$ having no cycles such that $K \subset \cup{ }_{i=1}^{l} W^{u}\left(\Lambda_{i}\right)$. Then if $\overline{W^{u}}\left(\Lambda_{i}\right) \cap W^{s}\left(\Lambda_{j}\right) \neq \varnothing\left(\right.$ or $\overline{W^{u}}\left(\Lambda_{i}\right) \cap$ $\hat{W}^{s}\left(\Lambda_{j}\right) \neq \varnothing$, if $i=j$ ) there exists a sequence $\Lambda_{j}=\Lambda_{j_{0}}, \ldots, \Lambda_{j_{k}}=\Lambda_{i}$ such that $\hat{W}^{u}\left(\Lambda_{j_{\alpha}}\right) \cap \hat{W}^{s}\left(\Lambda_{j_{\alpha-1}}\right) \neq \varnothing$ for $1 \leqslant \alpha \leqslant k$.

Proof. Lemma (1.3) implies that $\overline{W^{u}}\left(\Lambda_{i}\right) \cap \hat{W}^{s}\left(\Lambda_{j_{0}}\right) \neq \varnothing$. Let $x \in \overline{W^{u}}\left(\Lambda_{i}\right) \cap$ $\hat{W}^{s}\left(\Lambda_{j_{0}}\right)$. Since $K \subset \cup_{i=1}^{l} W^{u}\left(\Lambda_{i}\right)$, there is $1 \leqslant j_{1} \leqslant l$ such that $x \in W^{u}\left(\Lambda_{j_{1}}\right)$. Then $x \in \hat{W}^{u}\left(\Lambda_{j_{1}}\right) \cap \hat{W}^{s}\left(\Lambda_{j_{0}}\right)$. But $x \in \overline{W^{u}}\left(\Lambda_{i}\right) \cap \hat{W}^{u}\left(\Lambda_{j_{1}}\right)$ and so, by Lemma (1.3)(ii), we have $W^{u}\left(\Lambda_{i}\right) \cap W^{s}\left(\Lambda_{j_{1}}\right) \neq \varnothing$. Now assume $\Lambda_{j_{1}}, \ldots, \Lambda_{j_{p}}$ are defined for $p<l$ such that $\Lambda_{j_{\alpha}} \neq \Lambda_{i}$ for $1 \leqslant \alpha<p, \hat{W}^{u}\left(\Lambda_{j_{\alpha}}\right) \cap \hat{W}^{s}\left(\Lambda_{j_{\alpha-1}}\right) \neq \varnothing$ for $1 \leqslant \alpha \leqslant p$ and $\overline{W^{u}}\left(\Lambda_{i}\right) \cap \hat{W}^{s}\left(\Lambda_{j_{p}}\right) \neq \varnothing$. If $j_{p} \neq i$ we can obtain $\Lambda_{j_{p+1}}$ by the same process used to get $\Lambda_{j_{1}}$. Notice that the assumption of no cycles implies that $\Lambda_{j_{\alpha}} \neq \Lambda_{j_{\beta}}$ if $\alpha \neq \beta$. Since $1 \leqslant j_{\alpha} \leqslant l$ we must have $j_{\alpha}=i$ for some $\alpha$ which proves the lemma.

(1.5) Lemma. Let $\Lambda$ be an isolated set for $f$ and $x \in K$. If $\alpha(x) \cap \Lambda \neq \varnothing$ but $x \notin W^{u}(\Lambda)$, then

(i) $\alpha(x) \cap \hat{W}^{s}(\Lambda) \neq \varnothing$

(ii) $\alpha(x) \cap \hat{W}^{u}(\Lambda) \neq \varnothing$.

Proof. (i) Let $U$ be a compact neighborhood of $\Lambda$ such that $\bigcap_{n \in Z} f^{n}(U)=\Lambda$ and let $D^{s}=D^{s}(\Lambda, U)$ be a proper fundamental domain for $W_{\text {loc }}^{s}(\Lambda, U)$. It suffices to prove that given a neighborhood $V$ of $D^{s}$ such that $V \cap \Lambda=\varnothing$, we have $\vartheta_{-}(x) \cap V \neq \varnothing$. By Proposition (1.2) we know that $U^{\prime}=W_{\text {loc }}^{u}(\Lambda, U) \cup \vartheta_{+} V$ is a neighborhood of $\Lambda$. Since $\alpha(x) \cap \Lambda \neq \varnothing$, there exists $k>0$ such that $f^{-k}(x) \in U^{\prime}$. But $x \notin W^{u}(\Lambda)$, thus $f^{-k}(x) \notin W_{\text {loc }}^{u}(\Lambda, U)$ and so $f^{-k}(x) \in f^{n}(V)$ for some $n>0$, which implies $\theta_{-}(x) \cap V \neq \varnothing$.

(ii) Let $U$ be as above such that $x \notin U$. Since $f\left(W_{\text {loc }}^{s}(\Lambda, U)\right) \subset W_{\text {loc }}^{s}(\Lambda, U)$ we have $\theta_{-}(x) \cap W_{\text {loc }}^{s}(\Lambda, U) \neq \varnothing$. Let $x_{k}=f^{-n_{k}}(x) \in U$, with $n_{k} \rightarrow \infty$ as $k \rightarrow \infty$, such that $x_{k} \rightarrow z \in \Lambda$. Since $x_{k} \notin W_{\text {loc }}^{s}(\Lambda, U)$ we have $\theta_{+}\left(x_{k}\right) \not \subset U$. For each $k>0$, let $m_{k} \geqslant 0$ be such that $f^{m}\left(x_{k}\right) \in U$ for $0 \leqslant m \leqslant m_{k}$ but $f^{m_{k+1}}\left(x_{k}\right) \notin U$. Then $y_{k}=$ $f^{m_{k}}\left(x_{k}\right) \in\left(U-f^{-1}(U)\right)$ and $m_{k}-n_{k}<0$, that is $y_{k} \in \mathcal{O}_{-}(x)$ for, if not, we would have $0 \leqslant n_{k} \leqslant m_{k}$ and so $f^{n_{k}}\left(x_{k}\right)=x \in U$, which is a contradiction. We state now that $m_{k} \rightarrow \infty$ as $k \rightarrow \infty$. Indeed, given $N \in \mathbf{Z}^{+}$, there is $k_{0}>0$ such that $x_{k} \in$ $\cap{ }_{n>0}^{N} f^{-n}(U)$ for every $k \geqslant k_{0}$, since $x_{k}$ converges to $\Lambda$. Then $f^{n}\left(x_{k}\right) \in U$ for $0 \leqslant \underline{n} \leqslant N$ and so $m_{k} \geqslant N$ for every $k \geqslant k_{0}$. We can assume that $y_{k}$ converges to $y \in \overline{U-f^{-1}(U)}$ and we state that $y \in \hat{W}^{u}(\Lambda)$. It suffices to prove that $y \in$ $W^{u}(\Lambda)$, since $\Lambda \subset$ int $f^{-1}(U)$. If $y \notin W^{u}(\Lambda)$, there exists $j>0$ such that $f^{-j}(y) \notin$ $U$. Thus, there exists $k_{0}>0$ such that for every $k \geqslant k_{0}$ we have $m_{k}-j>0$ and $f^{m_{k}-j}\left(x_{k}\right)=f^{-j}\left(y_{k}\right) \notin U$, which is a contradiction since $0<m_{k}-j \leqslant m_{k}$.

From this lemma we obtain immediately the following result. 
(1.6) Corollary. If $\left\{\Lambda_{1}, \ldots, \Lambda_{l}\right\}$ is a family of disjoint isolated sets for $f$ such that $\alpha(x) \subset \cup_{i=1}^{l} \Lambda_{i}$ for every $x \in K$, then $\alpha(x) \subset \Lambda_{i}$ for some $i=1, \ldots, l$, that is, $K=\cup_{i=1}^{l} W^{u}\left(\Lambda_{i}\right)$.

In the proof of the Filtration Lemma which follows, we will need the following lemma that appears in [9].

(1.7) LeMmA. Let $F$ be a compact $f$-invariant subset of $K$ and $Q$ be a compact neighborhood of $F$ such that $\bigcap_{n \geqslant 0} f^{n}(Q)=F$. Then there is a compact neighborhood $V$ of $F$ such that $V \subset Q$ and $f(V) \subset$ int $V$.

(1.8) Filtration Lemma. If $\left\{\Lambda_{1}, \ldots, \Lambda_{l}\right\}$ is a family of disjoint isolated sets for $f$ having no clycles such that $\alpha(x) \subset \cup{ }_{i=1}^{l} \Lambda_{i}$ for every $x \in K$, then

(a) there exists a filtration for the family, that is, there are compact sets $\varnothing=K_{0} \subset$ $K_{1} \subset \cdots \subset K_{l}=K$ such that $f\left(K_{i}\right) \subset$ int $K_{i}$ and $\cap_{n \in Z} f^{n}\left(K_{i}-K_{i-1}\right)=\Lambda_{i}$.

(b) $\Omega(f) \subset \cap_{i=1}^{l} \Lambda_{i}$.

Proof. (a) We define the following relation on $\left\{\Lambda_{i}\right\}_{i=1}^{l}: \Lambda_{i} \leqslant \Lambda_{j}$ if there is a sequence $\Lambda_{j}=\Lambda_{j_{0}}, \ldots, \Lambda_{j_{k}}=\Lambda_{i}$ such that $\hat{W}^{u}\left(\Lambda_{j_{\alpha-1}}\right) \cap \hat{W}^{s}\left(\Lambda_{j_{\alpha}}\right) \neq \varnothing$ for $1 \leqslant \alpha<$ $k$. By the assumption of no cycles this relation is a partial ordering. It induces a total ordering $\Lambda_{1}<\Lambda_{2}<\cdots<\Lambda_{l}$ of the family where $\Lambda_{i}<\Lambda_{j}$ if $\Lambda_{i}<\Lambda_{j}$ but $\Lambda_{i} \neq \Lambda_{j}$. We define $K_{j}$ by induction. Let $K_{0}=\varnothing$ and assume we have defined compact sets $\varnothing=K_{0} \subset K_{1} \subset \cdots \subset K_{j}$ for $j<l$ such that

(i) $f\left(K_{i}\right) \subset$ int $K_{i}$,

(ii) $\cap_{n \in \mathbf{Z}} f^{n}\left(K_{i}-K_{i-1}\right)=\Lambda_{i}$ for $0<i \leqslant j$.

Let $F=\cup_{i<j+1} W^{u}\left(\Lambda_{i}\right)$. We claim that $F$ is closed and $F \cap \Lambda_{k}=\varnothing$ for $k>j+$ 1. Indeed let $\left(x_{n}\right)$ be a sequence in $F$ converging to $x \in K$. We can assume that for every $n>0$ we have $x_{n} \in W^{u}\left(\Lambda_{i}\right)$ for some fixed $i<j+1$. From Corollary (1.6) we know that there exists $1 \leqslant k \leqslant l$ such that $\alpha(x) \subset \Lambda_{k}$, that is, $x \in W^{u}\left(\Lambda_{k}\right)$. Then $x \in \overline{W^{u}}\left(\Lambda_{i}\right) \cap W^{u}\left(\Lambda_{k}\right)$. By Lemma (1.4) we conclude that $\Lambda_{k}<\Lambda_{i}$ and so $k<i \leqslant j+1$, that is, $x \in F$. Clearly $F \cap \Lambda_{k}=\varnothing$ for $k>j+1$.

Now let $Q \subset K$ be a compact neighborhood of $F$ such that $Q \cap\left(\cup_{i>j+1}^{l} \Lambda_{i}\right)=$ $\varnothing$. Thus $\bigcap_{n \geqslant 0} f^{n}(Q)=F$ for if $x \in \cap_{n>0} f^{n}(Q)$, then $f^{-n}(x) \in Q$ for every $n \geqslant 0$, that is, $\theta_{-}(x) \subset Q$, and therefore $\alpha(x) \subset Q$. Thus $\alpha(x) \subset \Lambda_{i}$ for some $i<j+1$, so $x \in \cup_{i<j+1} W^{u}\left(\Lambda_{i}\right)=F$. Since $F$ is invariant by $f$ we can use Lemma (1.7) and obtain a compact neighborhood $V_{j} \subset Q$ of $F$ such that $f\left(V_{j}\right) \subset$ $\operatorname{int}\left(V_{j}\right)$. Let $K_{j+1}=K_{j} \cup V_{j}$. Then we have $f\left(K_{j+1}\right) \subset$ int $K_{j+1}$ and we need only show that $\bigcap_{n \in \mathbf{Z}} f^{n}\left(K_{j+1}-K_{j}\right)=\Lambda_{j+1}$. First we observe that

$$
S=\bigcap_{n \in \mathbf{Z}} f^{n}\left(K_{j+1}-K_{j}\right)
$$

is closed for if $x_{k} \in S$ is a sequence converging to $x \in K$, then $f^{n}(x) \in K_{j+1}$ for every $n \in \mathbf{Z}$ since $K_{j+1}$ is compact. Suppose, to achieve a contradiction, that there exists $n \in Z$ such that $f^{n}(x) \in K_{j}$. Then $f^{n+1}(x) \in \operatorname{int}\left(K_{j}\right)$ and thus $f^{n+1}\left(x_{k}\right) \in$ int $K_{j}$ for large $k$, which contradicts the fact that $x_{k} \in S$. Thus $\alpha(x) \subset S$ for every $x \in S$, so $\alpha(x) \subset \Lambda_{j+1}$ for every $x \in S$, that is, $S \subset W^{u}\left(\Lambda_{j+1}\right)$. Similarly $\omega(x) \subset S$ for every $x \in S$. Thus using the dual of Lemma (1.5) we can conclude that 
$S \subset W^{s}\left(\Lambda_{j+1}\right)$. Then $S \subset W^{u}\left(\Lambda_{j+1}\right) \cap W^{s}\left(\Lambda_{j+1}\right)=\Lambda_{j+1}$. Now we observe that by Corollary (1.6), $K=\cup_{i=1}^{l} W^{u}\left(\Lambda_{i}\right)$ and so $K_{l}=K$.

(b) It suffices to prove that if $y \in K-\cup_{i=1}^{l} \Lambda_{i}$, then there exist $y^{\prime} \in \mathcal{O}(y)$ and a neighborhood $V$ of $y^{\prime}$ such that $f^{n}(V) \cap V=\varnothing$ for every $n>0$. Choose $\Lambda_{j}$ such that $\alpha(y) \subset \Lambda_{j} \subset$ (int $\left.K_{j}\right)-K_{j-1}$. Take $y_{1} \in \mathcal{O}(y)$ such that $\mathcal{O}_{-}\left(y_{1}\right) \subset K_{j}-K_{j-1}$. Since $y_{1} \notin \Lambda_{j}$, there exists a number $n_{0}>0$ such that $y_{2}=f^{n_{0}}\left(y_{1}\right) \notin K_{j}-K_{j-1}$. But $\theta_{+}\left(y_{1}\right) \subset K_{j}$ and thus $f^{n_{0}}\left(y_{1}\right) \in K_{j-1}$. Let $m_{0} \geqslant 0$ be such that $f^{-m}\left(y_{2}\right) \in K_{j-1}$ for $0 \leqslant m \leqslant m_{0}$ but $f^{-\left(m_{0}+1\right)}\left(y_{2}\right) \notin K_{j-1}$. Then $y^{\prime}=f^{-m_{0}}\left(y_{2}\right) \in K_{j}-f\left(K_{j-1}\right)$. Take a neighborhood $W \subset$ int $K_{j-1}$ of $f\left(K_{j-1}\right)$ and a neighborhood $V$ of $y^{\prime}$ such that $W \cap V=\varnothing$ and $f(V) \subset W$. Thus we have $f^{n}(V) \subset f^{n-1}(W) \subset W$ for every $n>0$ and therefore $V \cap f^{n}(V)=\varnothing$ for $n>0$.

(1.9) LEMMA. If $\left\{\Lambda_{1}, \ldots, \Lambda_{l}\right\}$ is a family of disjoint isolated sets for $f$ having no cycles, and if $x \in K$ satisfies $\alpha(x) \subset \cup_{i=1}^{l} W^{u}\left(\Lambda_{i}\right)$ then $x \in \cup_{i=1}^{l} W^{u}\left(\Lambda_{i}\right)$.

Proof. We will show that if $\alpha(x) \subset \cup_{i=1}^{l} W^{u}\left(\Lambda_{i}\right)$ but $x \notin \cup{ }_{i=1}^{l} W^{u}\left(\Lambda_{i}\right)$, then we can get a cycle. Choose $\Lambda_{i_{1}}, 1 \leqslant i_{1} \leqslant l$ such that $\alpha(x) \cap \Lambda_{i_{1}} \neq \varnothing$. Since $\alpha(x) \subset \Lambda_{i_{1}}$, from Lemma (1.5)(i), we have $\alpha(x) \cap \hat{W}^{s}\left(\Lambda_{i_{1}}\right) \neq \varnothing$. But by assumption, $\alpha(x) \subset \cup{ }_{i=1}^{l} W^{u}\left(\Lambda_{i}\right)$, so there exists $1 \leqslant i_{2} \leqslant l$ such that $\hat{W}^{u}\left(\Lambda_{i_{2}}\right) \cap \hat{W}^{s}\left(\Lambda_{i_{1}}\right)$ $\neq \varnothing$ and $\alpha(x) \cap \Lambda_{i_{2}} \neq \varnothing$. If $i_{2}=i_{1}$ we have a cycle. If not, we repeat the process and obtain $1 \leqslant i_{3} \leqslant l$ such that $\hat{W}^{u}\left(\Lambda_{i_{3}}\right) \cap \hat{W}^{s}\left(\Lambda_{i_{2}}\right) \neq \varnothing$ and $\alpha(x) \cap \Lambda_{i_{3}} \neq \varnothing$. Continuing as above, since there are only finitely many sets $\Lambda_{i}$, we eventually get a cycle.

(1.10) Lemma. Let $x \in K$. If $y \in \alpha(x)$, then given $\varepsilon>0$ there is a sequence $y=y_{0}, y_{1}, \ldots, y_{k}=y$ in $\alpha(x)$ such that $d\left(f\left(y_{j-1}\right), y_{j}\right)<\varepsilon$ for $1<j<k$.

Proof. Given $\varepsilon>0$, let $0<\delta<\varepsilon / 2$ such that if $p, q \in K$ and $d(p, q)<\delta$, then $d(f(p), f(q)) \leqslant \varepsilon / 2$. Since $f^{-n}(x)$ converges to $\alpha(x)$ as $n \rightarrow \infty$, there is $n_{0}>0$ such that $d\left(f^{-n}(x), \alpha(x)\right)<\delta, \forall n \geqslant n_{0}$. On the other hand, since $y \in \alpha(x)$ there are $n_{1}$, $k \geqslant 0$ such that $n_{1}-k \geqslant n_{0}$,

$$
d\left(f^{-n_{1}}(x), y\right)<\delta \text { and } d\left(f^{-n_{1}+k}(x), y\right)<\delta .
$$

Let $x^{\prime}=f^{-n_{1}}(x)$. Since $n_{1}-k \geqslant n_{0}$, if $1 \leqslant j \leqslant k$ then

$$
d\left(f^{j}\left(x^{\prime}\right), \alpha(x)\right)=d\left(f^{-n_{1}+j}(x), \alpha(x)\right)<\delta .
$$

Thus there exists $y_{j} \in \alpha(x)$ such that

$$
d\left(f^{j}\left(x^{\prime}\right), y_{j}\right)=d\left(f^{j}\left(x^{\prime}\right), \alpha(x)\right)<\delta,
$$

so

$$
d\left(f^{j+1}\left(x^{\prime}\right), f\left(y_{j}\right)\right)<\varepsilon / 2 .
$$

Then putting $y_{0}=y_{k}=y$ we get, for $1 \leqslant j \leqslant k$,

$$
d\left(f\left(y_{j-1}\right), y_{j}\right) \leqslant d\left(f\left(y_{j-1}\right), f^{j}\left(x^{\prime}\right)\right)+d\left(f^{j}\left(x^{\prime}\right), y_{j}\right)<\varepsilon / 2+\delta<\varepsilon
$$

proving the lemma.

(1.11) Lemma. Let $\left(\Sigma_{n}\right)_{n \in \mathbf{Z}^{+}}$be a family of compact subsets of $K$, invariant by $f$ such that if $n \geqslant m$ then $\Sigma_{n} \subset \Sigma_{m}$. For each $n \in \mathbf{Z}^{+}$, let $x_{n} \in \Sigma_{n}$ and $y_{n} \in \alpha\left(x_{n}\right)$ be 
such that $y_{n}$ converges to $y \in \Sigma=\bigcap_{n \in \mathbf{Z}^{+}} \Sigma_{n}$. Then, given $\varepsilon>0$, there is a sequence $y=\bar{y}_{0}, \bar{y}_{1}, \ldots, \bar{y}_{k}=y$ in $\Sigma$ such that $d\left(f\left(\bar{y}_{j-1}\right), \bar{y}_{j}\right)<\varepsilon$ for $1 \leqslant j \leqslant k$.

Proof. Given $\varepsilon>0$, choose $0<\delta<\varepsilon / 3$ such that if $p, q \in K$ and $d(p, q)<\delta$, then $d(f(p), f(q))<\varepsilon / 3$. Take $n_{0} \in \mathbf{Z}^{+}$such that if $x \in \Sigma_{n_{0}}$, then there exists $y^{\prime} \in \Sigma$ such that $d\left(x, y^{\prime}\right)<\delta$. Take $n>0$ such that $y_{n} \in \alpha\left(x_{n}\right) \subset \Sigma_{n_{0}}$ and $d\left(y, y_{n}\right)$ $<\delta$. From Lemma (1.10) we know that there is a sequence $y_{n}=z_{0}, z_{1}, \ldots, z_{k}=y_{n}$ in $\alpha\left(x_{n}\right) \subset \Sigma_{n_{0}}$ such that $d\left(f\left(z_{j-1}\right), z_{j}\right)<\varepsilon / 3$ for $1 \leqslant j<k$. Let $\bar{y}_{0}=\bar{y}_{k}=y$ and $\bar{y}_{j} \in \Sigma$ be such that $d\left(\bar{y}_{j}, z_{j}\right)<\delta$ for $1 \leqslant j \leqslant k$. Then $d\left(f\left(\bar{y}_{j-1}\right), \bar{y}_{j}\right) \leqslant$ $d\left(f\left(\bar{y}_{j-1}\right), f\left(z_{j-1}\right)\right)+d\left(f\left(z_{j-1}\right), z_{j}\right)+d\left(z_{j}, \bar{y}_{j}\right)<\varepsilon$ for $1 \leqslant j \leqslant k$, which proves the lemma.

Now we may proceed to the main theorem of this section. First we give a definition.

Definition. The Birkhoff center of $f, c(f)$, is the closure of the set of points in $K$ whose orbits are recurrent in the past as well as in the future. That is, $c(f)$ is the closure of the set $\{x \in K \mid x \in \alpha(x) \cap \omega(x)\}$.

(1.12) LeMMA. If $\Lambda \subset K$ is a compact set, invariant under $f$, such that $\Omega(f \mid \Lambda)=\Lambda$, then $\Lambda \subset c(f)$.

Proof. For each $n>0$ let $A_{n}^{+}=\left\{y \in \Lambda \mid d\left(f^{k}(y), y\right)<1 / n\right.$ for some $\left.k>0\right\}$ and $A_{n}^{-}=\left\{y \in \Lambda \mid d\left(f^{-k}(y), y\right)<1 / n\right.$, for some $\left.k>0\right\}$.

Clearly $A_{n}^{-}$and $A_{n}^{+}$are open and dense subsets of $\Lambda$, so $A_{n}=A_{n}^{-} \cap A_{n}^{+}$is an open and dense subset of $\Lambda$ for each $n>0$. Thus, since $\Lambda$ is a complete metric space, $A=\cap_{n>0} A_{n}$ is a dense subset of $\Lambda$, that is $\bar{A}=\Lambda$. But if $x \in A$ then $x \in \alpha(x) \cap \omega(x)$. Thus $\Lambda=\bar{A} \subset c(f)$.

Corollary. If $x \in \alpha(x)$, then $x \in c(f)$.

Proof. $\alpha(x)$ is a compact invariant set such that $\Omega(f \mid \alpha(x))=\alpha(x)$.

THEOREM A. If $c(f)$ is an isolated set for $f$ and admits a decomposition $c(f)=\Lambda_{1}$

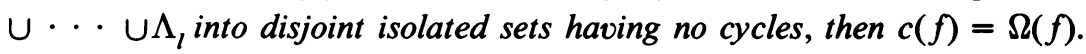

Proof. From Lemma (1.8) (filtration), it suffices to prove that $\alpha(x) \subset c(f)$ for every $x \in K$. Suppose, on the contrary, that there exists $x \in K$ such that $\alpha(x) \not \subset$ $c(f)$. Let $\mathscr{F}$ be the family of compact $f$-invariant subsets $\Sigma$ of $K$, such that there is an $x \in \Sigma$ with $\alpha(x) \not \subset c(f)$. We order $\mathcal{F}$ by inclusion. Now suppose for the moment that $\mathscr{F}$ possesses a minimal $\Sigma$. Then take $x \in \Sigma$ such that $\alpha(x) \not \subset c(f)$. From the corollary of Lemma (1.12) we have that $x \notin \alpha(x)$. So $\alpha(x)$ is a proper subset of $\Sigma$. Since $\alpha(x)$ is a compact $f$-invariant set and $\Sigma$ is a minimal set we conclude that $\alpha(x)$ is not in $\mathscr{F}$, that is, $\alpha(x) \subset \cup_{i=1}^{l} W^{u}\left(\Lambda_{i}\right)$. Thus from Lemma (1.9) we have $\alpha(x) \subset c(f)$, which is a contradiction. Thus to prove the theorem, it suffices to prove the following lemma.

LEMMA. $\mathcal{F}$ possesses a minimal $\Sigma$.

Proof. Let $\tilde{\tilde{F}}=\left\{\Sigma_{\beta}, \beta \in \mathscr{B}\right\}$ be any subfamily of $\mathscr{F}$ totally ordered by inclusion. Let $\Sigma=\bigcap_{\beta \in \mathscr{B}} \Sigma_{\beta}$. We need to prove that $\Sigma \in \mathscr{F}$. If $\Sigma \notin \mathscr{F}$, then 
$\alpha(x) \subset c(f)$ for every $x \in \Sigma$. Thus by Lemma (1.8) the family $\left\{\Lambda_{1} \cap \Sigma, \ldots, \Lambda_{l} \cap\right.$ $\Sigma\}$ admits a filtration and $\Omega(f \mid \Sigma) \subset \cup{ }_{i=1}^{l} \Lambda_{i} \cap \Sigma$. For each $\beta \in \mathscr{B}$, take $x_{\beta} \in \Sigma_{\beta}$ such that $\alpha\left(x_{\beta}\right) \not \subset c(f)$. We know that $\alpha\left(x_{\beta}\right) \cap\left(\cup_{i=1}^{l} \Lambda_{i}\right) \neq \varnothing$ for every $\beta \in \mathscr{B}$. Thus we can suppose, without loss of generality, that $\alpha\left(x_{\beta}\right) \cap \Lambda_{1} \neq \varnothing$ for every $\beta \in \mathscr{B}$. Let $U$ be a compact neighborhood of $\Lambda_{1}$ such that $\cap_{n \in \mathbf{Z}} f^{n}(U)=\Lambda_{1}$ and $U \cap\left(\cup_{i=2}^{l} \Lambda_{i}\right)=\varnothing$. Since $\alpha\left(x_{\beta}\right) \cap \Lambda_{1} \neq \varnothing$ but $\alpha\left(x_{\beta}\right) \not \Lambda_{1}$, we conclude from Lemma (1.5) that $\alpha\left(x_{\beta}\right) \cap D^{s} \neq \varnothing$, where $D^{s}=D^{s}\left(\Lambda_{1}, U\right) \subset U$ is a proper fundamental domain for $W_{\text {loc }}^{s}\left(\Lambda_{1}\right)$. For each $\beta \in \mathscr{B}$, choose $y_{\beta} \in \alpha\left(x_{\beta}\right) \cap D^{s}$. Let us take a subsequence $y_{\beta_{n}}$ such that $y_{\beta_{n}}$ converges to $y \in \Sigma \cap D^{s}$. Since $y \notin c(f)$ (recall that $y \in D^{s}$ and $D^{s} \cap c(f)=\varnothing$ ) and $\Sigma$ admits a filtration, there is a compact set $K^{\prime} \subset \Sigma$ and a point $y^{\prime} \in \mathcal{O}(y)$ such that $f\left(K^{\prime}\right) \subset$ int $K^{\prime}$ and $y^{\prime} \in K^{\prime}-$ $f\left(K^{\prime}\right)$. Let $W \subset K^{\prime}$ be an open set in $\Sigma$ such that $y^{\prime} \notin W$ and $f\left(K^{\prime}\right) \subset W$. Since $f\left(K^{\prime}\right)$ is compact, there is an $\varepsilon>0$ such that if $z \in \Sigma$ and $d\left(z, z^{\prime}\right)<\varepsilon$ for some $z^{\prime} \in f\left(K^{\prime}\right)$, then $z \in W$. From Lemma (1.11) we know that there exists a sequence $y^{\prime}=y_{0}, y_{1}, \ldots, y_{k}=y^{\prime}$ in $\Sigma$ such that $d\left(f\left(y_{j-1}\right), y_{j}\right)<\varepsilon$ for $1 \leqslant j<k$. We claim that $y_{j} \in W$ for $1 \leqslant j \leqslant k$. Indeed, since $f\left(y_{0}\right)=f\left(y^{\prime}\right) \in f\left(K^{\prime}\right)$ and $d\left(f\left(y_{0}\right), y_{1}\right)<\varepsilon$ we conclude that $y_{1} \in W \subset K^{\prime}$. Let us suppose that we have proved that $y_{j} \in W$ for $1 \leqslant j \leqslant k$. Then $f\left(y_{j}\right) \in f\left(K^{\prime}\right)$ and $d\left(f\left(y_{j}\right), y_{j+1}\right)<\varepsilon$, so $y_{j+1} \in W$. Thus $y^{\prime} \in$ $f\left(K^{\prime}\right)$, which is a contradiction. Thus $\Sigma \in \mathscr{F}$, proving the lemma.

We have concluded the proof of Theorem A. We will use it in the next section to prove our main result, Theorem $B$.

2. Hyperbolic Birkhoff centers. In this section we will consider a diffeomorphism of a compact manifold $M$. We will make use of the notation already introduced in $\S 1$ together with the concepts of hyperbolic sets and their invariant manifolds. With the help of Theorem A and well-known results, we will prove Theorem B. We observe that from [4], hyperbolic sets with local product structure are isolated in the sense of $\$ 1$, which enable us to make use of theorem $A$ in this context.

Throughout, $f$ is assumed to be a $C^{r}$-diffemorphism, $r \geqslant 1$, of a compact $C^{\infty}$ manifold without boundary. A compact set $\Lambda \subset M$ is hyperbolic for $f$ if $\Lambda$ is invariant under $f$, that is, $f(\Lambda)=\Lambda$, and $T f$ leaves invariant a continuous splitting $T_{\Lambda} M=E^{s} \oplus E^{u}$ expanding $E^{u}$ and contracting $E^{s}$. That is,

$$
\begin{aligned}
& |T f(v)| \leqslant \tau|v| \quad \text { if } v \in E^{s}, \\
& |T f(v)| \leqslant \tau^{-1}|v| \quad \text { if } v \in E^{u},
\end{aligned}
$$

for some constant $\tau, 0<\tau<1$, and some Riemannian metric on $M$. This Riemannian metric is said to be adapted to $\Lambda$.

Through each point $p$ of $\Lambda$, pass two smooth invariant manifolds, $W^{s}(p)$ and $W^{u}(p)$, tangent to $E_{p}^{s}$ and $E_{p}^{u}$, respectively. These two invariant manifolds are called the stable manifold and the unstable manifold of $p$, respectively [3]. Let us define

$$
\begin{aligned}
& W_{\varepsilon}^{s}(p)=\left\{y \in M \mid d\left(f^{n}(p), f^{n}(y)\right)<\varepsilon \text { for } n>0\right\}, \\
& W_{\varepsilon}^{u}(p)=\left\{y \in M \mid d\left(f^{-n}(p), f^{-n}(y)\right)<\varepsilon \text { for } n \geq 0\right\},
\end{aligned}
$$

where $d$ is the topological metric on $M$ induced by a Riemannian metric adapted to 
$\Lambda$. Then, $W^{s}(p)=\cup_{n \geqslant 0} f^{-n}\left(W_{\varepsilon}^{s}(p)\right)$ and $W^{u}(p)=\cup_{n>0} f^{n}\left(W_{\varepsilon}^{u}(p)\right)$, Recall that $W_{e}^{s}(p)$ (respectively $W_{e}^{u}(p)$ ) is an imbedded smooth manifold called the local stable (respectively unstable) manifold of "size $\varepsilon$ " of $p$. The union $W^{u}(\Lambda)=$ $\cup_{p \in \Lambda} W^{u}(p)$ is called the unstable manifold of $\Lambda$, and similarly $W^{s}(\Lambda)=$ $\cup_{p \in \Lambda} W^{s}(p)$ is called the stable manifold of $\Lambda$.

Definition. A hyperbolic set $\Lambda$ has a local product structure if, for some $\varepsilon>0$,

$$
W_{\varepsilon}^{u}(p) \cap W_{\varepsilon}^{s}(q) \subset \Lambda
$$

for all $p, q \in \Lambda$.

According to results in [4], if $\Lambda$ is a hyperbolic set having a local product structure, then $\Lambda$ is an isolated set for $f$ in the sense of $\S 1$, and $W^{u}(\Lambda)=\{x \in$ $M \mid \alpha(x) \subset \Lambda\}$. That is, the unstable manifold of $\Lambda$ coincides with the unstable space associated to the isolated set $\Lambda$ as in $\S 1$; similarly for $W^{s}(\Lambda)$.

We denote by $\operatorname{Diff}^{r}(M)$ the set of $C^{r}$ diffeomorphisms of $M$ with the uniform $C^{r}$ topology, $r \geqslant 1$. We call $f \in \operatorname{Diff}^{r}(M) \Omega$-stable if there exists a neighborhood $N$ of $f$ in $\operatorname{Diff}^{r}(M)$ such that for each $g \in N, g \mid \Omega(g)$ is topologically conjugate to $f \mid \Omega(f)$.

Definition. We say that $f$ satisfies Axiom A if

(a) $\Omega=\Omega(f)$ has a hyperbolic structure,

(b) the set of periodic points is dense in $\Omega$.

In [5], Newhouse proved that if $\bar{P}$ is hyperbolic, then $\bar{P}$ has a decomposition, $\bar{P}=\Lambda_{1} \cup \Lambda_{2} \cup \cdots \cup \Lambda_{l}$ into disjoint hyperbolic sets having local product structure. From the observation above, $\left\{\Lambda_{1}, \ldots, \Lambda_{l}\right\}$ is a disjoint family of isolated sets.

Next we present a lemma which we will use in the proof of Theorem B. This lemma, first communicated by Anosov, can be proved using a stronger result showed by Bowen [1]. Newhouse gives, in [5], a proof for the case $\Lambda=L^{-}$.

(2.1) Anosov's Closing Lemma. If $\Lambda$ is a hyperbolic set for $f$, then $\bar{P} \supset \Omega(f \mid \Lambda)$.

We can now prove Theorem $B$.

THeOREM B. If $c(f)$ is hyperbolic and has no cycles, then $f$ satisfies Axiom A and has no cycles. In particular, $f$ is $\Omega$-stable.

Proof. Since $\bar{P} \subset c(f), \bar{P}$ is hyperbolic. Thus $\bar{P}$ has a decomposition into disjoint isolated sets. So, by Theorem $\mathrm{A}$, it suffices to prove that $c(f)=\bar{P}$. But this fact is a direct consequence of Anosov's Closing Lemma and the fact that $c(f)=\Omega(f \mid c(f))$. In particular, from [9], $f$ is $\Omega$-stable.

We recall that $f$ is called a Kupka-Smale diffeomorphism if $f$ satisfies

(a) the periodic points of $f$ are hyperbolic,

(b) the transversality condition.

If, in addition, $\Omega(f)$ is finite, $f$ is said to be a Morse-Smale diffeomorphism.

As an immediate consequence of Theorem B, we have the following result.

(2.2) Corollary. If $f$ is a Kupka-Smale diffeomorphism, and $c(f)$ is finite, then $f$ is in fact a Morse-Smale diffeomorphism.

We observe that Theorem B is not true if we replace $c(f)$ by the center of minimal sets, i.e., the closure of the minimal sets of $f$. An example is the time-one 
map of Cherry's flow [2]. In this case the center of minimal sets is composed of the two fixed points of the diffeomorphism, which are hyperbolic without cycles, but does not coincide with the nonwandering set. In particular, this diffeomorphism is not $\Omega$-stable.

3. A partial converse. In this section we prove Theorem $\mathbf{C}$, which is a partial converse to our main result. We also give a characterization of $\Omega$-stability when $c(f)$ is finite.

We first introduce the concept of $\bar{P}$-stability. Following the notation established in the preceding sections, $\bar{P}=\overline{\operatorname{Per}(f)}$ will denote the closure of the set of periodic points of $f$.

We say that $f \in \operatorname{Diff}^{\prime}(M), r \geqslant 1$, is $\bar{P}$-stable if there exists a neighborhood $N$ of $f$ in $\operatorname{Diff}^{r}(M)$ such that for each $g \in N, g \mid \overline{\operatorname{Per}(g)}$ is topologically conjugate to $f \mid \overline{\operatorname{Per}(f)}$.

Definition. Let $f \in \operatorname{Diff}^{r}(M)$ with $\bar{P}=\overline{\operatorname{Per}(f)}$ hyperbolic. An $n$-cycle $(n \geqslant 0)$ on $\bar{P}$ is a sequence of basic sets $\Lambda_{0}, \ldots, \Lambda_{n}, \Lambda_{n+1}$, such that $\hat{W}^{u}\left(\Lambda_{i}\right) \cap \hat{W}^{s}\left(\Lambda_{i+1}\right)$ $\neq \varnothing, \Lambda_{0}=\Lambda_{n+1}$ and otherwise $\Lambda_{j} \neq \Lambda_{k}$ for $j \neq k$. Here a basic set $\Lambda$ is a hyperbolic set with local product structure such that $f \mid \Lambda$ is topologically transitive and the periodic points are dense in $\Lambda$.

(3.1) TheOREM. If $f$ is $\bar{P}$-stable and $\bar{P}=\overline{\operatorname{Per}(f)}$ is hyperbolic, then the decomposition of $\bar{P}$ into basic sets has the no cycle property.

Proof. We first prove the following assertion: if $f$ is $\bar{P}$-stable and there is an $n$-cycle on $\bar{P}$, then there exists $g$ near $f$ in $\operatorname{Diff}^{r}(M)$ such that $\overline{\operatorname{Per}(g)}$ is hyperbolic for $g$ and has an $(n-1)$-cycle.

From the stability of hyperbolic sets, and the $\bar{P}$-stability of $f$, we have that there exists a neighborhood $V$ of $\bar{P}$ such that for $g$ near $f$ in $\operatorname{Diff}^{r}(M), \overline{\operatorname{Per}(g)}$ is contained in $V$ and is hyperbolic for $g$ [3].

Let $\Lambda_{0}, \ldots, \Lambda_{n}, \Lambda_{n+1}$ be an $n$-cycle on $\bar{P}=\overline{\operatorname{Per}(f)}$. From

$$
W^{s}\left(\Lambda_{i}\right)=\bigcup_{x \in \Lambda_{i}} W^{s}(x) \text { and } W^{u}\left(\Lambda_{i}\right)=\bigcup_{x \in \Lambda_{i}} W^{u}(x),
$$

we have $W^{u}\left(x_{i}\right) \cap W^{s}\left(y_{i+1}\right) \neq \varnothing$ for some $x_{i}, y_{i} \in \Lambda_{i}$ and $0 \leqslant i \leqslant n$. We claim that at least for one of the indices $j, \operatorname{dim} W^{u}\left(x_{j}\right)+\operatorname{dim} W^{s}\left(y_{j+1}\right) \geqslant \operatorname{dim} M$. For otherwise, if $r=\sum_{i=0}^{n}\left(\operatorname{dim} W^{u}\left(x_{i}\right)+\operatorname{dim} W^{s}\left(y_{i+1}\right)\right)$ then $r<n \operatorname{dim} M$. But since $\Lambda_{n+1}=\Lambda_{0}$ and $\operatorname{dim} W^{u}\left(x_{i}\right)+\operatorname{dim} W^{s}\left(y_{i}\right)=\operatorname{dim} M$, we have $r=n \operatorname{dim} M$, thus reaching a contradiction. Let $y \in W^{u}\left(x_{j}\right) \cap W^{s}\left(y_{j+1}\right)$, the index $j$ chosen as above. Clearly $y \notin \bar{P}$. Thus after a small perturbation of $f$ near $y$ we get $f_{1}$ near $f$ in $\operatorname{Diff}^{r}(M)$ so that, from the above observations, $\overline{\operatorname{Per}\left(f_{1}\right)}=\bar{P}, f_{1}|\bar{P}=f| \bar{P}, W^{u}\left(x_{j}, f_{1}\right)$ and $W^{s}\left(y_{j+1}, f_{1}\right)$ have a point of transversal intersection, and $W^{u}\left(x_{i}, f_{1}\right) \cap$ $W^{s}\left(y_{i+1}, f_{1}\right) \neq \varnothing$ for $0 \leqslant i \leqslant n$. We may assume that $x_{j}, y_{j+1}$ are periodic points of $f$ (and thus of $f_{1}$ ). Now, $\left.f_{1}\right|_{\Lambda_{j+1}}=\left.f\right|_{\Lambda_{j+1}}$ is topologically transitive and so we may further assume $y_{j+1}=x_{j+1}$. Let $z \in W^{u}\left(x_{j+1}, f_{1}\right) \cap W^{s}\left(y_{j+2}, f_{1}\right)$. Since we have assumed that $f$ is $\bar{P}$-stable, we have that $z \notin \operatorname{Per}\left(f_{1}\right)=\bar{P}$. Since closure $W^{u}\left(x_{j}, f_{1}\right)$ $\supset W^{u}\left(x_{j+1}, f_{1}\right)$, we claim that by a small local perturbation of $f_{1}$ near $z$ we get $g$ 
near $f$ in $\operatorname{Diff}^{r}(M)$ with $\overline{\operatorname{Per}(g)}=\bar{P}, g|\bar{P}=f| \bar{P}$ and $W^{u}\left(x_{j}, g\right) \cap W^{s}\left(y_{j+2}, g\right) \neq \varnothing$, thus producing an $(n-1)$-cycle in $\bar{P}=\overline{\operatorname{Per}(g)}$ and hence proving our assertion. To prove this, we use the following facts which are immediate consequences of the $\lambda$-lemma in [8]. Let $\delta>0$ and $F$ be a small neighborhood of $W_{\delta}^{u}\left(x_{j+1}, f_{1}\right)$.

(i) Consider $x \in W^{s}\left(x_{j+1}, f_{1}\right) \cap F$ and a disc $D \subset F$ through $x$ of dimension equal to $\operatorname{dim} W^{u}\left(x_{j+1}, f_{1}\right)$ and transverse to $W^{s}\left(x_{j+1}, f_{1}\right)$ at $x$. Then given $\varepsilon>0$, there exists $n>0$ and a disc $D_{n} \subset f_{1}^{n}(D)$ such that $D_{n}$ is $\varepsilon-C^{r}$ near $W_{\delta}^{u}\left(x_{j+1}, f_{1}\right)$ and $f_{1}^{-j}\left(D^{n}\right) \subset F$ for $0 \leqslant j \leqslant n$.

(ii) If $z \in W_{\delta}^{u}\left(x_{j+1}, f_{1}\right)$ and $V \subset F$ is a sufficiently small neighborhood of $z$, then if $y \in V \cap f_{1}^{k}(V)$ for some $k>0$, there exists $0 \leqslant j<k$ such that $f_{1}^{-j}(y) \notin F$.

We now prove our claim. Let $W_{\delta}^{u}\left(x_{j+1}, f_{1}\right)$ and $F$ be as above. Take $z \in$ $W_{\delta}^{u}\left(x_{j+1}, f_{1}\right) \cap W^{s}\left(y_{j+2}, f_{1}\right)$ and $x \in F$ a point of transversal intersection between $W^{u}\left(x_{j}, f_{1}\right)$ and $W^{s}\left(x_{j+1}, f_{1}\right)$. Let $V$ be a small neighborhood of $z$ such that $V$ satisfies (ii), $\theta(x) \cap V=\varnothing$ and $\vartheta_{+}(z) \cap V=\{z\}$. Let $D$ be a small disc in $W^{u}\left(x_{j}, f_{1}\right) \cap F$ transversal to $W^{s}\left(x_{j+1}, f_{1}\right)$ such that $D \cap W^{s}\left(x_{j+1}, f_{1}\right)=\{x\}$. Since $\theta(x) \cap V=\varnothing$ we can take $D$ such that $\theta_{-}(D) \cap V=\varnothing$. Using (i) we can obtain $n>0$ and $y \in D_{n} \subset f_{1}^{n}(D)$ sufficiently near $z$, so that there exists a diffeomorphism $h$ of $M$, which carries $y$ to $z$, coincides with the identity map outside $V$ and is $\varepsilon-C^{r}$ near the identity map. We then take $g=f_{1} \circ h$. From (i) and (ii) we have that $f_{1}^{-k}(y) \notin V$ for every $k>0$ and thus $y \in W^{u}\left(x_{j}, g\right)$. From the choice of $V$ we also have that $z \in W^{s}\left(y_{j+2}, g\right)$. Since $g(y)=z$ we conclude that $z \in W^{u}\left(x_{j}, g\right) \cap$ $W^{s}\left(y_{j+2}, g\right)$.

The proof of the theorem goes now by induction on the length $n$ of the cycle and the fact that if $\bar{P}$ has a zero-cycle then $f$ is not $\bar{P}$-stable. For, as above, after a small local perturbation of $f$, say to $f_{1}, W^{u}\left(x_{0}, f_{1}\right)$ and $W^{s}\left(x_{0}, f_{1}\right)$ have a point of transversal intersection outside $\bar{P}$, that is a transversal homoclinic point. From [10], this point is accummulated by periodic points, and so $f$ and $f_{1}$ are not $\bar{P}$-conjugate and thus $f$ is not $\bar{P}$-stable. This finishes the proof of the theorem.

From Lemma (2.1), the above theorem and Theorem B we get

THEOREM C. If $c(f)$ is hyperbolic and $f$ is $\Omega$-stable, then $c(f)$ has the no cycle property. In particular, $c(f)=\Omega(f)$.

As a consequence of this theorem and Theorem $B$ we get

(3.2) Corollary. Let $f \in \operatorname{Diff}^{r}(M)$ and $c(f)$ be finite. Then $f$ is $\Omega$-stable if and only if $\Omega(f)=c(f)$ is hyperbolic and has the no cycle property.

\section{REFERENCES}

1. R. Bowen, Periodic points and measures for Axiom A diffeomorphisms, Trans. Amer. Math. Soc. 154 (1971), 377-397.

2. T. Cherry, Analytic quasi-periodic curves of discontinuous type on a torus, Proc. London Math. Soc. 44 (1938), 175-215.

3. M. Hirsch and C. Pugh, Stable manifolds and hyperbolic sets, Proc. Sympos. Pure Math., vol. 14, Amer. Math. Soc., Providence, R.I., 1970, pp. 133-163.

4. M. Hirsch, J. Palis, C. Pugh and M. Shub, Neighborhoods of hyperbolic sets, Invent. Math. 9 (1969/70), 121-134. 
5. S. Newhouse, Hyperbolic limit sets, Trans. Amer. Math. Soc. 167 (1972), 125-150.

6. L Lectures on dynamical systems (C.I.M.E. Summer Session in Dynamical Systems, Bressanone, Italy, June 1978).

7. J. Palis, A note on $\Omega$-stability, Proc. Sympos. Pure Math., vol. 14, Amer. Math. Soc., Providence, R.I., 1970, pp. 221-222.

8.

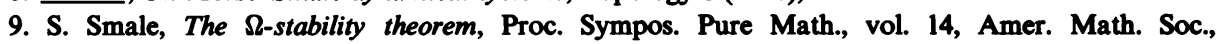
Providence, R.I., 1970, pp. 289-297.

10. Diffeomorphisms with many periodic points, Differential and Combinatorial Topology, Princeton Univ. Press, Princeton, N.J., 1975.

laboratório de Cálculo, Centro brasileiro de Pesquisas físicas, Rio de Janeiro, Brasil

Current address: Centro Brasileiro de Pesquisas Físicas, Av. Wenceslau Braz, 71-Fundos, 20.000-Rio de Janeiro, Brasil 